\title{
Competency Acquisition in Applied Knowledge Engineering
}

\author{
An Approach based on Learning from Errors
}

\author{
Vera G. Meister \\ Department of Business and Management \\ Brandenburg University of Applied Sciences \\ Brandenburg a.d.H., Germany \\ vera.meister@th-brandenburg.de
}

\begin{abstract}
The ability to model business knowledge is one of the core competences in the discipline of Information Systems. In addition, the modeling of business processes and structures has found wide acceptance in study programs for economic sciences. In addition to the knowledge of modeling paradigms, notations and tools, an effective acquisition of skills in this area also requires a deeper understanding of a professional domain, preferably from the point of view of a service provider or producer. Exercises that only rely on the analysis of existing business models or on the implementation of purely textual descriptions are not sufficient to establish a deeper understanding of the subject as well as an effective competence acquisition. The paper aims to show how a didactic concept, which takes up existing domain knowledge and explicitly focuses on a positive error culture, contributes significantly to the acquisition of competence in knowledge engineering by students.
\end{abstract}

Keywords: component; Business modeling, didactics of Information Systems, learning from errors, competence-oriented learning, domain knowledge

\section{INTRODUCTION}

Bridging the gap between business users and decisionmakers in companies and organizations on the one hand and IT developers and implementers on the other is considered the foremost task in the field of Information Systems (comp. [1]). Traditionally, the term business IT alignment is used, while recent developments in information and communication technology are more likely to take on the character of IT business enabling.

In this article, the following definition of the term knowledge is used: "Knowledge is a fluid mix of framed experience, values, contextual information, and expert insight ... It originates and is applied in the minds of knowers. In organizations, it often becomes embedded not only in documents or repositories but also in organizational routines, processes, practices, and norms. [2]"

Business knowledge in companies and organizations can thus be found as implicit knowledge in the minds of employees or in practiced business processes but can also be available explicitly in the form of descriptions, models and / or programs. It is typically highly fragmented and highly variable; it is distributed across departments, groups and individuals, and is often accessible only through human interpretation, like shown in [3]. Closing the proverbial gap between business and IT therefore means formally modeling this knowledge in such a way that it remains understandable to people (or only becomes so) and can be processed or evaluated by machines. According to [4], this is exactly what is central to knowledge engineering.

In the last ten years, an impressive portfolio of bridging technologies has been established for the (semi-) formal modeling of professional knowledge, which increasingly becomes object of teaching and learning in the fields of Information Systems, Business Administration and Computer Science. The present case study focuses on two compulsory elective modules, which are offered in the department of Business and Management at a university of applied sciences in the 4th semester of the bachelor's program Information Systems and in the 3rd semester of the master's program Business Administration. Both student groups have already acquired basic knowledge about modeling methods and notations, basic skills in the use of tools and applications as well as basic competences of system analysis, abstraction and reflection.

The subject of the mentioned courses is a bundle of standard-specific, practice-relevant modeling technologies that are to be applied and documented in the context of individual, practical projects. Students at universities of applied sciences of the above-mentioned middle and higher age groups already have practical experience from internships or previous or study-related gainful employment, so that a sufficiently profound expertise in an exemplary business domain can be assumed. The title "Applied Knowledge Engineering" (AKE) was chosen to distinguish the course from basic theoretical as well as highly technical aspects of knowledge engineering.

The following section first addresses the courses on AKE as a research topic and, in this context, explains the research question as well as the implemented research design. On the one hand there is the method of structured observation and reflection directly during the course and on the other hand a questionnaire-based, qualitative evaluation at the end of the course. The procedure details and the results are presented in 
Sections III and IV respectively. The work concludes with a conclusion and a brief critical reflection.

\section{RESEARCH TOPIC AND RESEARCH DESIGN}

The subject of this work is a series of AKE courses. In this section, first the content specification and the didactic concept of these courses are presented. Central didactic element is a complex, individual project on (semi-) formal business modeling. The aim of this paper is to prove that the errors and iteration steps that inevitably accompany these activities significantly promote the acquisition of competences.

\section{A. Specification of Course Content}

When selecting the bridging technologies for the AKE courses, emphasis was initially placed on graphically connoted, standard-specified notations with proximity to the subject-specific modeling. An important selection criterion was also the degree of diffusion and the relevance of a notation for Business Information System as a discipline. These requirements are met in an outstanding way by OMG's recently completed notation triad: BPMN, CMMN and DMN [5-7]. The letter sequence "MN" stands for "Model and Notation", which should make it clear that these are formally specified and at the same time graphically usable technologies. These notations allow the modeling of business processes, knowledge-intensive work and operational business decisions. To formally represent the vocabulary used in business modeling and prepare it for further use, the W3C standard SKOS has been included, which is based on RDF, the basic data structure for semantic modeling $[8,9]$. Finally, with RDF, it is possible to map semantic networks in general, and thus also arbitrary business structures and relations.

Table I gives an overview of the mentioned technologies, the addressed modeling objects as well as relevant information technology fields of application.

TABLE I. PORTFOLIO OF TECHNOLOGIES FOR AKE

\begin{tabular}{|l|c|c|}
\hline \multicolumn{1}{|c|}{ Modeling subject } & $\begin{array}{c}\text { Notation/ } \\
\text { Specification }\end{array}$ & Field of application \\
\hline Business processes & BPMN 2.0 (OMG) & Digitized processes \\
\hline $\begin{array}{l}\text { Knowledge intensive } \\
\text { case work }\end{array}$ & CMMN 1.1 (OMG) & $\begin{array}{c}\text { IT support of } \\
\text { knowledge work }\end{array}$ \\
\hline Operational decisions & DMN 1.1 (OMG) & Automated rules \\
\hline $\begin{array}{l}\text { Vocabularies and } \\
\text { thesauries }\end{array}$ & SKOS (W3C) & Big data analysis \\
\hline $\begin{array}{l}\text { Structures and } \\
\text { relations }\end{array}$ & RDF (W3C) & Artificial intelligence \\
\hline
\end{tabular}

\section{B. Didactic Setting}

In addition to the acquisition of knowledge about the technologies themselves as well as the acquisition of skills in tool-based modeling, the learning objectives of the courses under consideration include the following competence goals:

- Analyze and define problems of the real world,
- Communicate with stakeholders,

- Choose a suitable level of abstraction,

- Formalize concepts and entities of the real world and their relationships,

- Select appropriate notation or specification,

- Apply notations and specifications syntactically correct,

- Choose appropriate tools and use them properly,

- Critically reflect one's own work.

For this purpose, a didactic setting was developed iteratively, over several semesters, which provides a cycle for each technology in the portfolio. It consists of knowledge input, structured knowledge transfer, and knowledge application in the individual business domain accompanied by multi-channel coaching. Fig. 1 visualizes this concept in a compressed form.

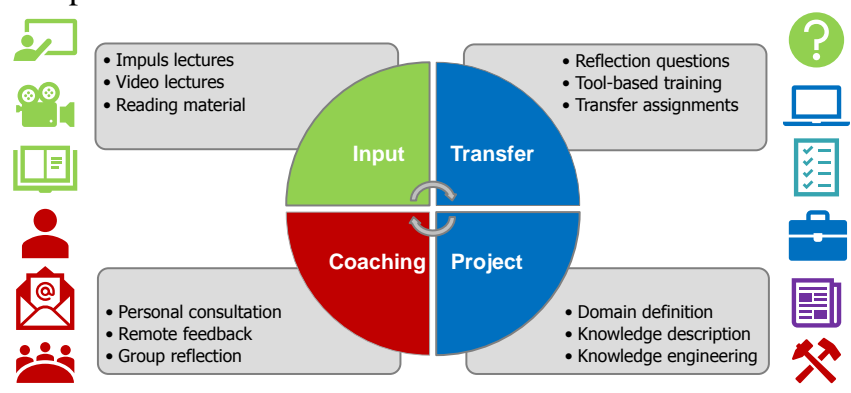

Figure 1. Didactic setting of the course AKE

\section{Learning from Errors}

As stated in [4], each model is only an approximation of reality, so modeling may potentially have to be considered as an infinite process. In addition, it is a cyclical process because newly discovered knowledge can lead to refinements, changes or completions. Finally, modeling requires an interpretation of the reality being modeled, which inherently includes error options.

It should be mentioned again that the course aims at the development of modeling competencies. On the other hand, the "objective" quality of the models created can be regarded as secondary. From these premises, the following implications were drawn:

1) In order to provide sufficient space for competence development, the process of knowledge acquisition according to [3] should be shortened as far as possible. This can be achieved by locating the domain to be modeled in the personal experiential space of every student. If students have no professional experience, leisure activities can also be used for modeling if the position of a service provider (eg the organizer of an event) is taken.

2) Errors in the process of modeling need to be seen as an opportunity for deeper understanding and development of better models rather than failure to meet a requirement. Individual modeling leads to individual errors. A positive error culture can be achieved by giving each student possibly several times - the opportunity to critically reflect on and discuss collegially his or her modeling drafts. 
The approach declared in point 2) is supported by Karl Poppers theory of learning from errors. In [10] he argues that errors (serious problems) break our expectation horizon, that is, our world-understanding framework, like a bomb, so that we are required to reconstruct it. These efforts, at best, lead to a new, higher-level world explanation horizon, which now integrates the problematic observations.

In [11] are discussed three professional competences of teachers regarding domain-specific errors of learners: (i) the awareness of possible types of errors, (ii) the application of appropriate measures or reactions, (iii) a constructional view on errors and their usage in teaching and learning processes. The latter has already been addressed above in point 2). Competence (ii) is reflected in the coaching part of each learning cycle (see Fig. 1), and error types will be discussed in Section III.

\section{Research Hypothesis and Research Methods}

The design implications derived and discussed in the previous subsection about the acquisition of competences in AKE should now be formulated in a research hypothesis: The possibility to model in one's own domain and thereby make individual errors contributes significantly to the learning success. For testing the hypothesis, two research methods were used. First, an observation was installed during the course, which implicitly identifies the types of errors encountered and the apparent increase in competence. Further details can be found in Section III. To cover the students' point of view, a qualitative-empirical method was used in a specific course instance. All active participants were asked to answer a series of mostly pre-structured questions. This procedure is described in Section IV.

\section{OBSERVATION DURING THE COURSE}

From the author's personal many years of experience in supervising complex student modeling projects, the following types of errors result: selection of an inappropriate abstraction level; incomplete analysis and description of structures, processes and relationships; neglection of restrictions or support options of tools; misuse of notational syntax; weak or incorrect implementation of notation logic and semantics; application of suboptimal patterns.

All these aspects are discussed in introductory lectures and illustrated with examples. However, it has repeatedly been shown that a transfer of knowledge and a deeper understanding can only be achieved through one's own practice and iterative reflection. The students are many-times informed and encouraged to acquire a positive culture of error. The framework for this is formed in the semester defined milestones, to which students submit work stalls of their modeling. As soon as possible, they receive written or oral feedback on their models.

For this, the following feedback rules are used: (i) Stylistic errors are marked in the respective element and referenced to the relevant style specification. (ii) Syntax errors often do not just affect one element. Here the relevant area is marked. Reference is made to syntactic rules and / or implications of the identified error. Since these implications often run counter to the modeling intent, the root cause of the error becomes visible. (iii) For all other, more complex errors (in logic, the level of abstraction, the technical stringency, etc.), the problems are discussed in several layers, ideally domain-specific. Possibly, solutions are proposed, but not fully executed.

The method of observation will be explained by means of a BPMN process model. Fig. 2 illustrates an excerpt from an early student submission with a series of marked errors and the corresponding comments. Fig. 3 shows an improved submission of the same modeling excerpt with visualization of the respectively corrected or revised aspects.

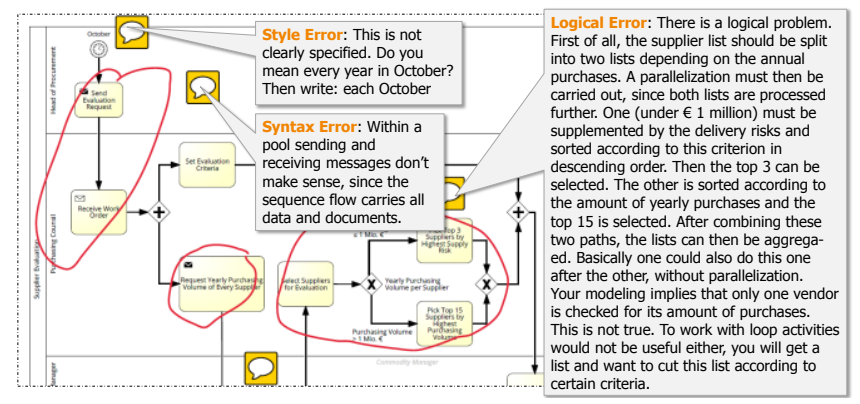

Figure 2. Student BPMN model with errors and comments (excerpt)

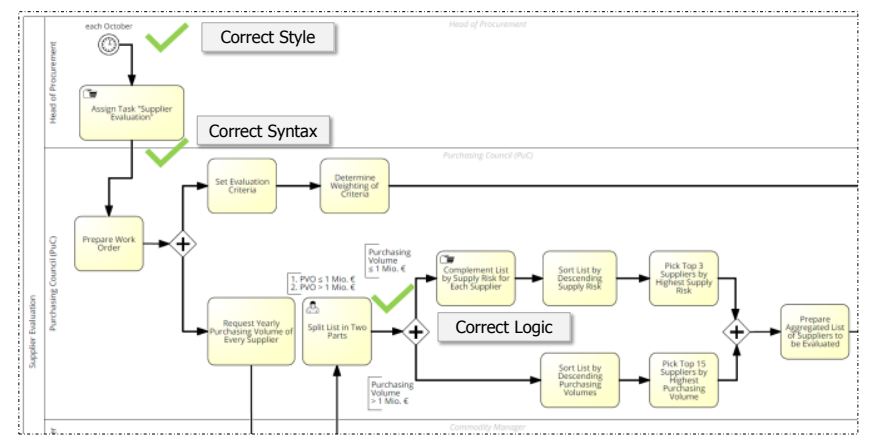

Figure 3. Final student BPMN model with corrections (excerpt)

It turns out that the correction of the stylistic error requires no own creativity. It is doubtful that such an error in the context of purely graphic modeling leads to a new construction of the explanatory horizon according to Karl Popper. It would be different if the process models also had to be implemented technically. In this case, such an error could also lead to execution errors. The modeling syntax was corrected by redesigning the task distribution and specification. Since this is the basic question of how participants communicate within a process, it can be assumed that significant new explanations must have been constructed here by the modeling student. This becomes even clearer with the logical error, which was cured by a completely new modeling pattern.

In sum, it can be expected that the student has developed a deeper understanding of the process in his or her domain, the modeling skills have generally improved, and that he or 
she has acquired a broader understanding of the process notation, its semantics, options and restrictions.

\section{Qualitative EVALUATION}

The research presented in this paper has the character of a case study in that it examines complex, elusive phenomena in their natural context (see [12]). It took place directly in the context of active teaching and is not embedded in a separate research project. The qualitative evaluation had to be limited to the participants of a specific course instance.

\section{A. Outline of the Survey}

The survey was conducted as part of a final presentation session at the end of the course. The written, paper-based questionnaire was completed anonymously by all active students $(\mathrm{N}=21)$. They were not explicitly informed about which items are subject to the special investigation. In addition to questions that explicitly address the research thesis presented in Subsection II.B, further items were queried. The survey was announced as a teaching evaluation with the goal of quality improvement.

Table II summarizes all items investigated, their coding structure and their relatedness to the research thesis.

TABLE II. DETAILS OF SURVEY OUTLINE

\begin{tabular}{|c|c|c|}
\hline Investigated item & Coding structure & $\begin{array}{c}\text { Relatedness to } \\
\text { thesis }\end{array}$ \\
\hline $\begin{array}{l}\text { self-assessment of knowledge } \\
\text { and skills at the beginning } \\
\text { and end of the course }\end{array}$ & $\begin{array}{l}\text { 6-step scale: } \\
\text { from not available } \\
\text { until excellent }\end{array}$ & $\begin{array}{l}\text { no direct } \\
\text { relatedness }\end{array}$ \\
\hline $\begin{array}{l}\text { frequency of use of } 4 \text { different } \\
\text { forms of learning or media }\end{array}$ & $\begin{array}{l}\text { 4-level scale for } \\
\text { each form or media: } \\
\text { never until often }\end{array}$ & no relatedness \\
\hline $\begin{array}{l}\text { helpfulness of the } 4 \text { different } \\
\text { forms of learning or media }\end{array}$ & ranking & no relatedness \\
\hline $\begin{array}{l}\text { possibly missed alternative } \\
\text { learning forms or media }\end{array}$ & open & no relatedness \\
\hline $\begin{array}{l}\text { personal feeling regarding the } \\
\text { approach "learning from } \\
\text { errors" }\end{array}$ & $\begin{array}{l}3 \text { predefined options: } \\
\text { unpleasant, unfami- } \\
\text { liar, challenging; } \\
1 \text { open option }\end{array}$ & $\begin{array}{l}\text { indirect } \\
\text { relatedness }\end{array}$ \\
\hline $\begin{array}{l}\text { Consent to the statements: } \\
\text { (i) The ability to make errors } \\
\text { and learn from them has } \\
\text { contributed significantly to } \\
\text { my learning success. } \\
\text { (ii) The ability to model in my } \\
\text { own domain of expertise has } \\
\text { contributed significantly to } \\
\text { my learning success. }\end{array}$ & $\begin{array}{l}\text { each 6-step scale: } \\
\text { do not agree to } \\
\text { agree completely }\end{array}$ & $\begin{array}{l}\text { direct } \\
\text { relatedness }\end{array}$ \\
\hline
\end{tabular}

\section{B. Evaluation of Results not Related to the Research Thesis}

The investigated items without direct relatedness to the research thesis are nevertheless of interest in the given context. On the one hand, they illustrate the self-assessment of students regarding their technical knowledge and their skills in AKE. On the other hand, they contribute to the general evaluation of the didactic setting presented in Subsection II.B. The question of possibly missing alternative forms of learning or media was answered only by five students. Wiki without HTML, mini-exercises, tool tutorials were each called once. Two mentions were attributed to frontal presentations of a modeling procedure, to transferred "without difficulty" to one's own domain.

In assessing the frequency of use and the helpfulness of the various forms or media of learning offered, the survey focused on the key didactic elements: classroom lectures, video lectures, e-mail feedback, and individualized consultations. Interestingly enough, the classroom lectures were used most frequently (median: often) and performed well in terms of helpfulness (median: most helpful). Regarding both, the usage rate and utility they were followed by e-mail feedback. Fig. 4 shows an average representation of the answers as a diagram.

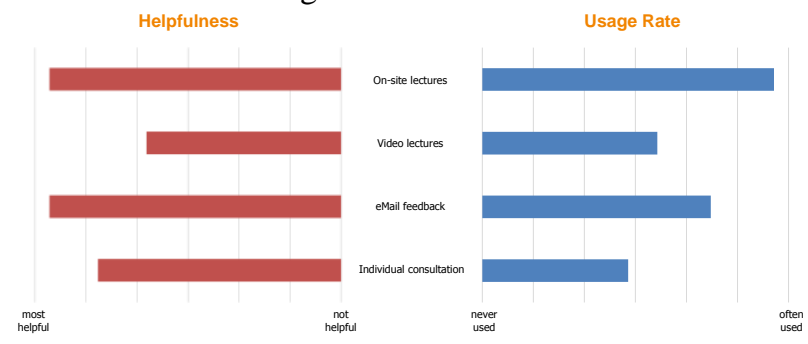

Figure 4. Frequency of use and usefulness of learning forms or media

The self-assessment of modeling knowledge and skills was differentiated according to business processes, business decisions, and business vocabularies. The results illustrate the fact that students already have some skills in modeling business processes from previous courses. In all aspects, students recognize a significant increase in their knowledge and skills. In the median view, the values regarding business processes increase by two levels, in the decision modeling by four levels, and in the modeling of vocabularies by three levels. Fig. 5 shows the results in an averaged representation.

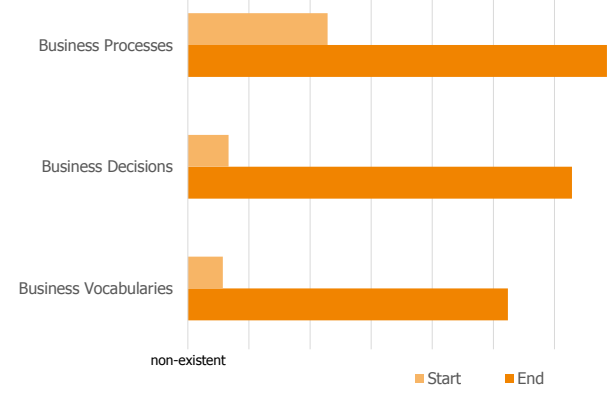

Figure 5. Students' self-assessment of their knowledge and skills

\section{Evaluation of Results Related to the Research Thesis}

For the question of personal perception of "learning from errors", the questionnaire provides three hard-coded answer options: (i) unpleasant, (ii) unfamiliar, (iii) challenging. The fourth option provided was a free text field. The latter was 
only used by one participant and marked as "too much effort". Assumed that students consider challenging and unfamiliar learning settings to be positive (eight in each case), this question was answered positively by $75 \%$ of the participants.

The most direct reference to the research thesis is the level of agreement to the statements that (i) modeling in an

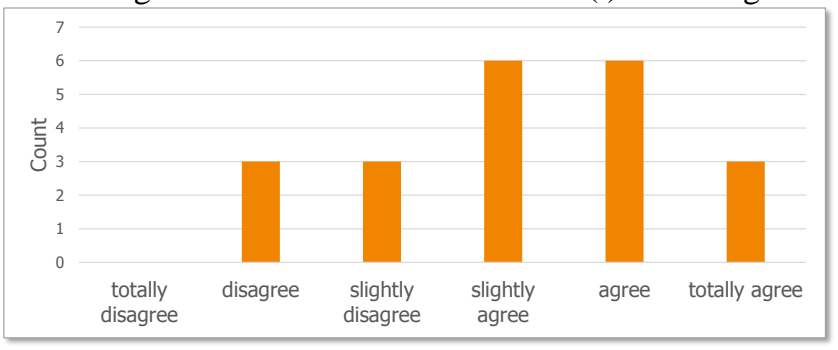

Figure 6. Agreement to the statement: Learning from my own errors has contributed significantly to my learning success

\section{CONCLUSION AND REFLECTION}

For students of Business Administration programs, a learning setting of the type described is unusual. They judged the course to be challenging and some too hard. All active students of the course achieved at least good results. In an oral feedback session, all them reported motivation gains resulting from personal expertise in the domain and intensive coaching. The acquisition of competence in this short period of time can be rated as above average, which is primarily due to the extensive individual modeling practice. The same applies to the efforts of the lecturer to give this differentiated feedback on a variety of models and development steps.

Both applied research methods, the structured observation and interaction during the course as well as the questionnaire-based qualitative evaluation at the end of the course support the thesis of a positive effect of the conceptual approaches: "learning from errors" and "modeling in one's own domain" to the desired learning success. Because of the small number of participants in the survey and the limited objectivity of the author being the responsible creator of the course, the results may not be transferable without further ado. On the other hand, complementary informal observations, e.g. from personal discussions and not systematically documented feedback sessions, rather support a confirmation of the thesis and thus suggest a further use of this didactic setting. individual domain and (ii) learning from own errors contribute significantly to the learning success. If one interprets the three upper values as agreement and the three lower values as rejection (in each case differentiated according to slightly, default and totally) in the six-step scale, the approval rate for both statements is 67\%. Fig. 6 and 7 show the distributions in detail.

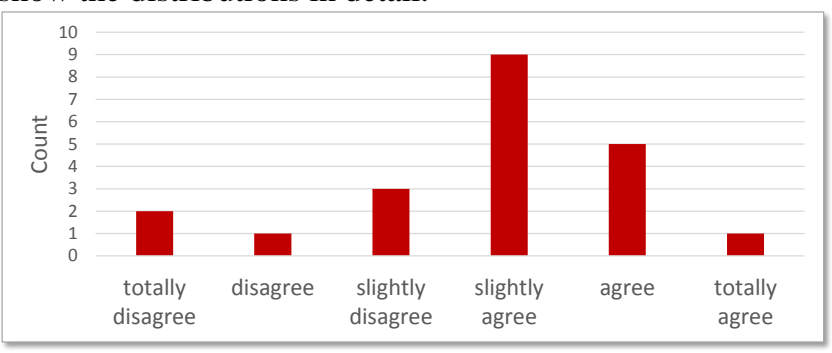

Figure 7. Agreement to the statement: modeling in my own domain has contributed significantly to my learning success

\section{REFERENCES}

[1] R. K. Rainer, B. Prince, and H. J. Watson, Management Information Systems, 3rd ed., Hoboken (NJ): Wiley Publishing, 2015.

[2] T. H. Davenport and L. Prusak, Working Knowledge: How Organizations Manage What They Know, Cambridge: Harvard Business Press, 2000, p.5.

[3] G. Probst, S. Raub, and K. Romhardt, Wissen managen, $6^{\text {th }}$ ed., Wiesbaden: Gabler, 2010, p. 28 and pp. 65f.

[4] R. Studer, R. Benjamin, and D. Fensel, "Knowledge Engineering Principles and Methods," Data \& knowledge engineering, vol. 25, $\mathrm{N}^{\circ}$ 1-2, 1998, pp.161-197.

[5] Object Management Group, Business Process Model and Notation 2.0, 2011, http://www.omg.org/spec/BPMN/2.0/.

[6] Object Management Group, Case Management Model and Notation 1.1, 2016, http://www.omg.org/spec/CMMN/1.1.

[7] Object Management Group, Decision Model and Notation 1.1, 2016, http://www.omg.org/spec/DMN/1.1/.

[8] World Wide Web Consortium, SKOS Simple Knowledge Organization System, 2009, https://www.w3.org/TR/skos-reference/.

[9] World Wide Web Consortium, RDF 1.1 Concepts and Abstract Syntax, 2014, https://www.w3.org/TR/skos-reference/.

[10] K. Popper, Objective Knowledge - An Evolutionary Approach, Oxford: Clarendon Press, 1972, pp.344f.

[11] J. Türling, J. Seifried, and E. Wuttke, "Teachers' Knowledge about Domain Specific Student Error,” in: E. Wuttke, J. Seifried (eds.), Learning from Errors at School and at Work, Opladen: Budrich, 2012, pp.90-102.

[12] T. Wilde and T. Hess, "Forschungsmethoden der Wirtschaftsinformatik," WIRTSCHAFTSINFORMATIK, vol. 49, $\mathrm{N}^{\mathrm{0}} 4,2007$, pp.280-287. 